\title{
A Convergent Approach to the Viability of the Dynamical Systems: The Cognitive Value of Complexity
}

\author{
Oleg Illiashenko ${ }^{1 *}$, Valeriy Mygal², Galyna $\mathrm{Mygal}^{3}$, Olga Protasenko ${ }^{4}$ \\ ${ }^{1}$ Department of Computer Systems, Networks and Cybersecurity, National Aerospace University "KhAI", Kharkiv 61074, \\ Ukraine \\ ${ }^{2}$ Department of Physics, National Aerospace University " KhAI ", Kharkiv6 1074, Ukraine \\ ${ }^{3}$ Department of Automobile and Transport Infrastructure, National Aerospace University " KhAI ", Kharkiv 61074, Ukraine \\ ${ }^{4}$ Department of Technologies and Life Safety, Simon Kuznets Kharkiv National University of Economics, Kharkiv 61166 , \\ Ukraine
}

Corresponding Author Email: o.illiashenko@khai.edu

https://doi.org/10.18280/ijsse.110612

Received: 9 January 2021

Accepted: 22 November 2021

\section{Keywords:}

safety, dynamic complexity, human factor, risks, cognitive aspects, modelling, signal structure, spatio-temporal signatures

\begin{abstract}
The integration of information and industrial technologies, digitalization and differentiation of sciences are accompanied by an increase in various types of complexity. This limits the capabilities of computer modelling, data mining, and predictive analytics. The increasing cognitive complexity of information flows and their diversity creates problems of safety, reliability and stability of the functioning of a complex dynamic system in extreme conditions. Here we show the possibility of cognitive visualization of signals of different nature through their geometrization in the form of a topological 3D model of functioning. Its projections are spatio-temporal signatures, the configurations of which reflect the dynamic, energetic and structural features of the model. An increase in the number of components of the signature configuration and its area under external influence indicates an increase in structural and functional complexity. Therefore, the signal structure can be analyzed in real time using complementary probabilistic and deterministic methods. A set of tools for the synthesis and analysis of 3D models has innovative potential for monitoring the functioning of elements of complex dynamic systems, risk management and predictive analytics.
\end{abstract}

\section{INTRODUCTION}

The integration of information and industrial technologies, and the digitalization of research, modelling and diagnostics have increased the complexity of data mining, risk management and predictive analytics [1]. The variety of processing methods, visualization and analysis of information increases the information complexity, which leads to the manifestation of cognitive aspects in the management of complex dynamic systems (CDS) in extreme conditions [2]. The consequence is the increasing complexity of the interaction of all elements of the VTS, including humans. Their consistency determines the safety, reliability and stability that determine the viability of the CDS. In extreme conditions, it also depends on thinking in complexity of a person (operator, dispatcher, etc.), who makes a quick decision $[3,4]$ (Figure 1).

The discrepancy between thinking in complexity and the complexity of the CDS functioning limits the control possibilities. Despite the further growth of computational capabilities, there is an asymmetry in the interconnections between formalised signs of CDS elements functioning. Therefore, the complexity of the dynamic structure of information flows and their diversity limits the possibilities of predictive analytics, modelling, data mining, etc.

An increase in static complexity under external influences is characteristic for all CDS elements. Moreover, their characteristic signs are not related to each other and considered in different subject areas. Analysis of the interconnections between different types of complexity in Figure 1 clockwise allows identifying the backbone factors that determine CDS functioning. Analysis counter clockwise allows identifying factors that destroy the system. The balance of these factors is characteristic of self-organised dynamic systems and phenomena, described using the principle of detailed equilibrium (dynamic balance) [5]. Therefore, the concept of complexity is interdisciplinary and common to all dynamic systems. Beer, Wiener, Prigogine and Haken developed various types of complexity [6-9]. All complexity types are manifested in the thinking in complexity $[3,10]$. Creative and critical thinking depends on information overload and human psychophysiological state. The consequence of this is an increase in risks (Figure 2). Their main reason is the dynamic complexity of processes (phenomena), which appears because of time delays in the interaction.

The variety of interactions in CDS is associated with:

(1) the nonlinearity of self-organised processes and their interconnectedness;

(2) lack of sufficient quantitative information on the processes dynamics;

(3) the variability of the character of dynamic processes in time and space. 


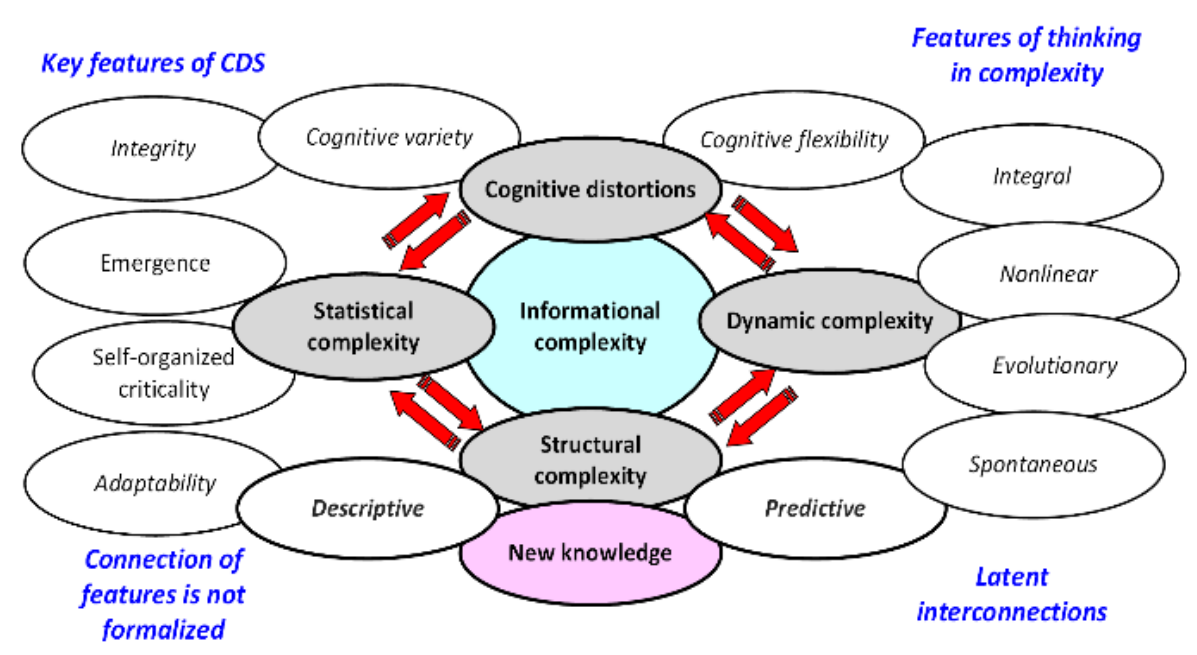

Figure 1. Key signs of CDS functioning and features of thinking in complexity

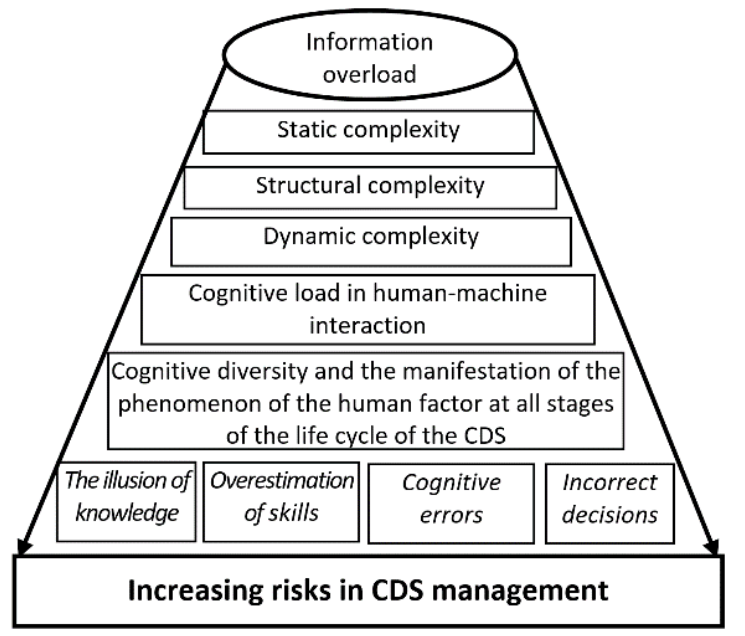

Figure 2. Causal relationship between information overload and increased risk

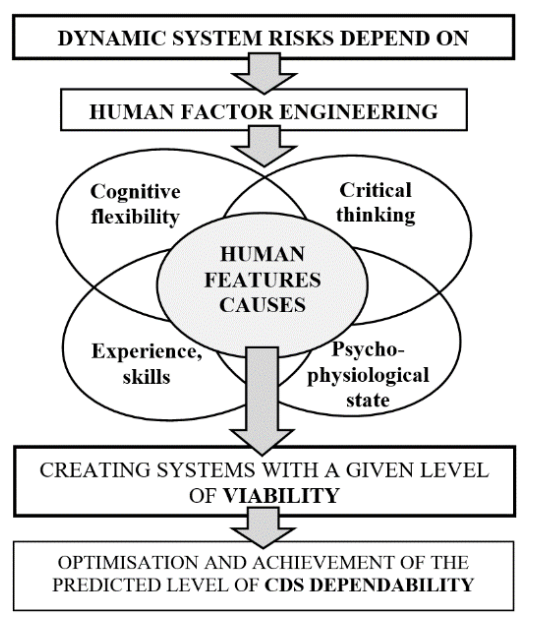

Figure 3. CDS risks associated with human factors

The cognitive diversity of relevant information sources leads to information overload (memory impairment, concentration, etc.), which negatively affects the decisions. The dynamic complexity of information sources and information transmission lines increase structural complexity and, accordingly, cognitive load. It leads to cognitive diversity and cognitive distortions in man-machine interaction [11, 12]. Dynamic complexity creates risks in CDS management.
Figure 2 shows the causal relationship between information overload and risks increasing during the CDS operation upon extreme conditions.

Improving CDS safety depends on taking into account the capabilities, cognitive, behavioural and psychophysiological person characteristics. Therefore, it is essential to assess the human factor's role as a key element in ensuring the CDS viability. It allows for solving problems of risk decreasing (Figure 3).

Accounting the cognitive aspects of CDS functioning upon extreme conditions is necessary to develop intelligent decision support and predictive analytics systems. Obviously, it determines the growing interest in human factor engineering and other interdisciplinary sciences. Their main task is to form an interdisciplinary vision of man-machine interaction features upon extreme conditions, the role of cognitive psychology, and cognitive ergonomics in ensuring safety culture [13].

The aim is cognitive visualisation of $3 \mathrm{D}$ models of the dynamic systems' functioning of different nature.

\section{INTERCONNECTION OF DIFFERENT TYPES OF COMPLEXITY AND ITS COGNITIVE VALUE}

Expert councils of engineers, ergonomists, programmers, physicists, biophysicists use the same terms, patterns, etc., the semantic definitions of which are different. Therefore, they sometimes do not understand each other. Different types of difficulty are affected by:

(1) an increase in the number of information sources (sensors, detectors, spectrometers, fractal biosignals, etc.), which increases the static complexity;

(2) the presence of spatial inhomogeneities in information sources (semiconductor sensors and other sensors) and its transmission media, which generate temporal inhomogeneities and increase dynamic complexity;

(3) the interaction of spatial and temporal inhomogeneities, which generates a latent structure of relationships.

As a result, the environment static complexity gives the increase of passing streams dynamic complexity, increasing the structural complexity. They all increase the cognitive load of man-machine interaction. It leads to the appearance of cognitive problems associated with different types of complexity (Figure 4). 


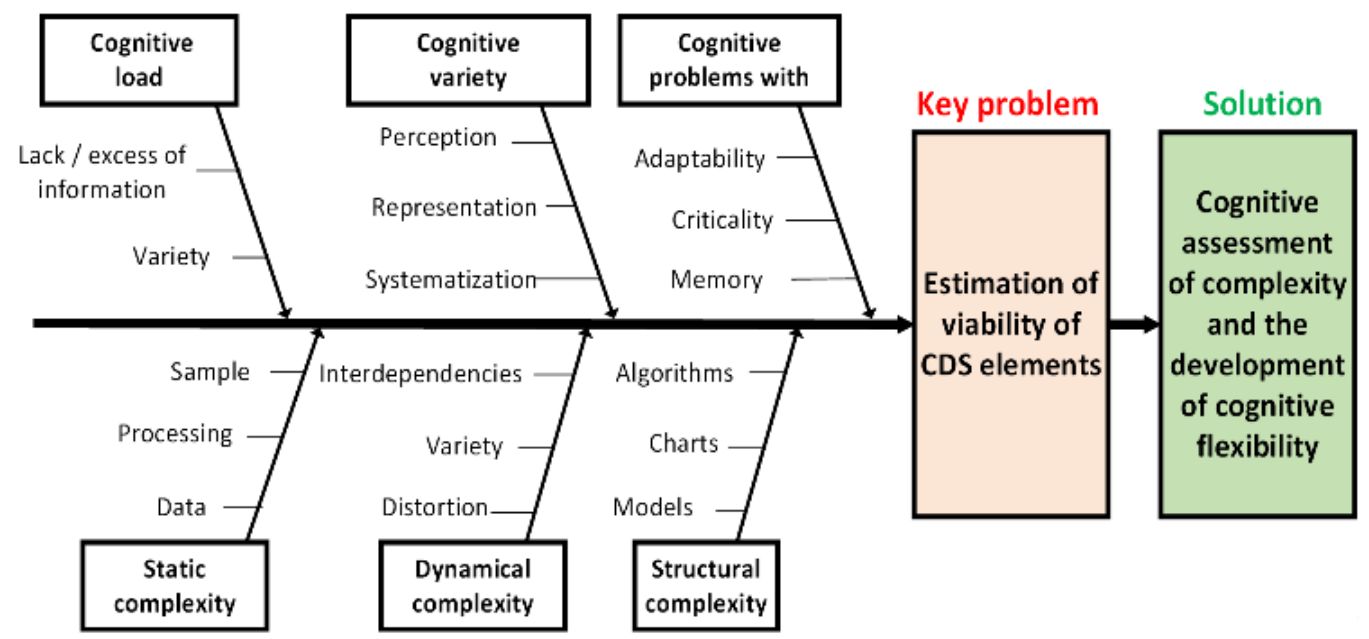

Figure 4. Cognitive aspects of different types of complexity

Making quick decisions requires cognitive flexibility. Its development is facilitated by transformational learning in the cognitive space [13], as well as the use of ergonomic laws of mutual adaptation and transformation $[14,15]$.

Therefore, identifying the latent structure of relationships in the CDS limits the increasing complexity of computer modelling, which is based on the principles of system dynamics $[16,17]$. The complexity of the synthesis of models increases the complexity of the analysis, which, in turn, complicates: the selection of relevant information sources, identification and classification of states, decision making in real-time.

The variety of explicit and latent heterogeneities of different nature and scale in sensors of different nature and information transmission environment manifests in the different types of complexity.

Latent connections between them limit the possibilities of modelling real processes and phenomena. Therefore, the development of technology and applied sciences constantly faces various physical limitations [18]. For instance, spectral analysis has simplified the identification and classification of atoms and molecules. However, their self-structuring on the nanoscale in smart materials is hidden and is manifested only in dynamics [19]. In continuous electromagnetic environments, structure-sensitive phenomena (induction, self-induction and mutual induction) are researched by statistical, deterministic and fractal methods [20] (Figure 5).

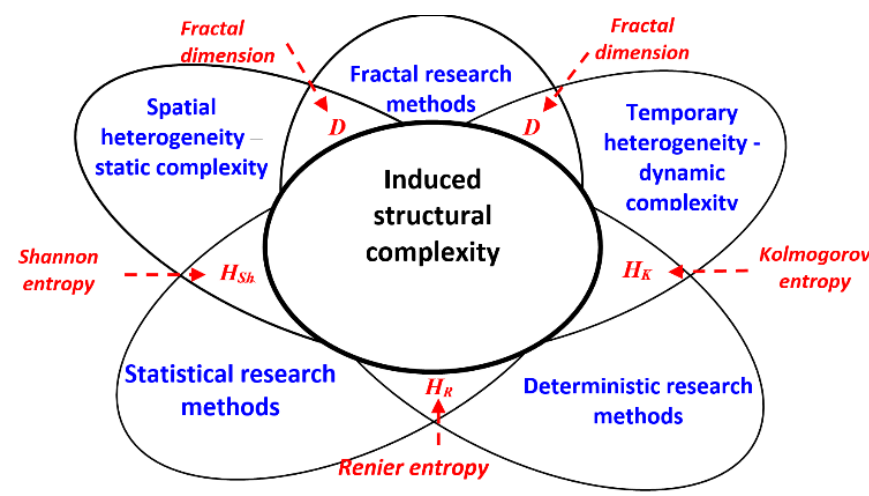

Figure 5. Methods for investigating structural complexity induced by inhomogeneities

In the statistical analysis of SDS, the entropies of Shannon, Kolmogorov, Ranier and fractal dimension are used [21].
From the systems analysis of Figure 5, it follows that induced structural complexity makes it possible to combine different methods of research and cognitive analysis. However, this requires identifying the induced order and balance of opposites that arise to counteract external influences. Dual nature of destabilising processes appears in this, and it is the same for all environments.

\section{DUALISM INDUCED BY COMPLEXITY}

The reason for most of the cognitive problems of manmachine-environment interaction is in the dualism of destabilising factors. On the one hand, they generate various local signal distortions that increase static and dynamic complexity. And their interconnection, in turn, limits the possibilities of modelling functioning in real-time. On the other hand, an intense destabilising factor leads to selforganised criticality [22], which induces new interconnections between spatial and temporal inhomogeneities.

In non-equilibrium processes, the principle of detailed balance is fulfilled, which reflects the natural balance of opposites. This balance underlies all evolutionary phenomena of nature, and information is hidden in spatial and temporal local features of functioning. Consequently, locally concentrated signal features are potential sources of information about the ordering and balance of opposites, the degree of which is an individual characteristic of sensors of different nature and information transmission medium. The key reason for cognitive problems is desynchronization of information flows (signals from sensors, detectors, and other information sources). The impact of destabilising factors leads to cognitive dissonance and cognitive distortions. At the same time, the person psychophysiological state affects the cognitive perception of complexity, diversity and instability.

\section{STRUCTURAL APPROACH TO STUDYING THE INDIVIDUALITY OF FUNCTIONING}

At the most fundamental level, modern physics (chemistry, biology, biophyschemistry, etc.) appears to us as a world of structures [23]. It is easier to analyse a large number of interconnected sources of information if their structures are similar. Understanding this point of view allowed us to assume that the spatio-temporal ordering of links between local 
distortions of the time series (fractal signal, information flow) contains latent information about the structure of induced interconnections.

Individuality and dynamic complexity limit the possibilities of digital modelling and cognitive computing. Simultaneously, the distribution of local distortions in a fractal signal contains information about opposite processes. Some processes contribute to the self-structuring of induced interconnections, while others destroy existing interconnections. That is why, to assess the viability of the information source (sensor, etc.), complementary extreme principles of natural science can be applied, which manifest in the space of dynamic events. We introduced this space to study the objects functioning individuality of animate and inanimate nature [24]. The cognitive value of the dynamic event space is that it can be justified based on:

(1) theories of dimensions and dynamic similarity, on the basis of which an interdisciplinary approach to the study of individuality has been implemented;

(2) variational principles of dynamics, on the basis of which a natural reconstruction of the topological 3D model of the functioning of a dynamic system based on the measured fractal signal was carried out;

(3) methodology of dynamic balance (equilibrium), which is based on the principles of biomimicry [25].

From the complementarity of the cognitive value substantiation of the space of dynamic events, it follows that morphologically different dynamic systems are functionally subordinate to the same principles and are described by the statistical laws of physics, biology, etc. Therefore, the manifestation of the detailed balance principle in the space of dynamic events and the complementarity of the dynamic's principles make it possible to model the functioning of CDS elements of different nature, including the human organism. Attention is drawn to the uniqueness and significance of natural science's extreme principles in researches [26-30]. In particular, for topological models of the functioning of dynamic systems, the following has a cognitive value:

(1) complementarity of the least action principles (Hamilton and A. Hertz) and the biomimicry principles;
(2) geometrization of the Jacobi principle of least action in the form of a geodesic curve;

(3) the energy interpretation of the Gauss principle of least compulsion and the theorem of E. Noether.

The interconnection of these principles with biomimicry underlies the ergonomic laws of mutual adaptation and transformation [14].

\section{COGNITIVE VISUALIZATION}

One-dimensional time series $\mathrm{X}(\mathrm{t})$ of different nature can be transformed into 2D and 3D models of functioning [31-33]. Fractal and multifractal digitized electrophysiological signals $\mathrm{V}(\mathrm{t})$ - (ECG, EEG, EOG, rheograms, etc.) are of the greatest cognitive value. The influence of external and internal factors on them is in the PhysioNet database [34]. Comparison of three methods of presentation and analysis of the dynamics of the EKG signal is shown in Figure 6.

From the comparative analysis, it follows that the greatest cognitive information is contained in the topological 3D model and its orthogonal projections. It turned out that the phase portrait as a sequence of dynamic states is a simplified model of the 1st order signature. 2nd order spatio-temporal signatures have cognitive value. Their configurations reflect the spatiotemporal interconnections of energy and structural components of the functioning cycle. The projections of the topological 3D functioning model are individual graphic images (signatures of the 1st and 2nd orders). Therefore, the creation of an atlas that is based on "PhysioNet" makes it possible to identify and classify the human functional state in real-time.

Their configurations reflect dynamic, energetic and structural features of functioning. Signature configurations reflect the natural decomposition of a time series (fractal signal) into opposite components. This made it possible to use in their analysis the principle of detailed equilibrium (dynamic balance), which underlies the phenomena of nature and biomimicry.

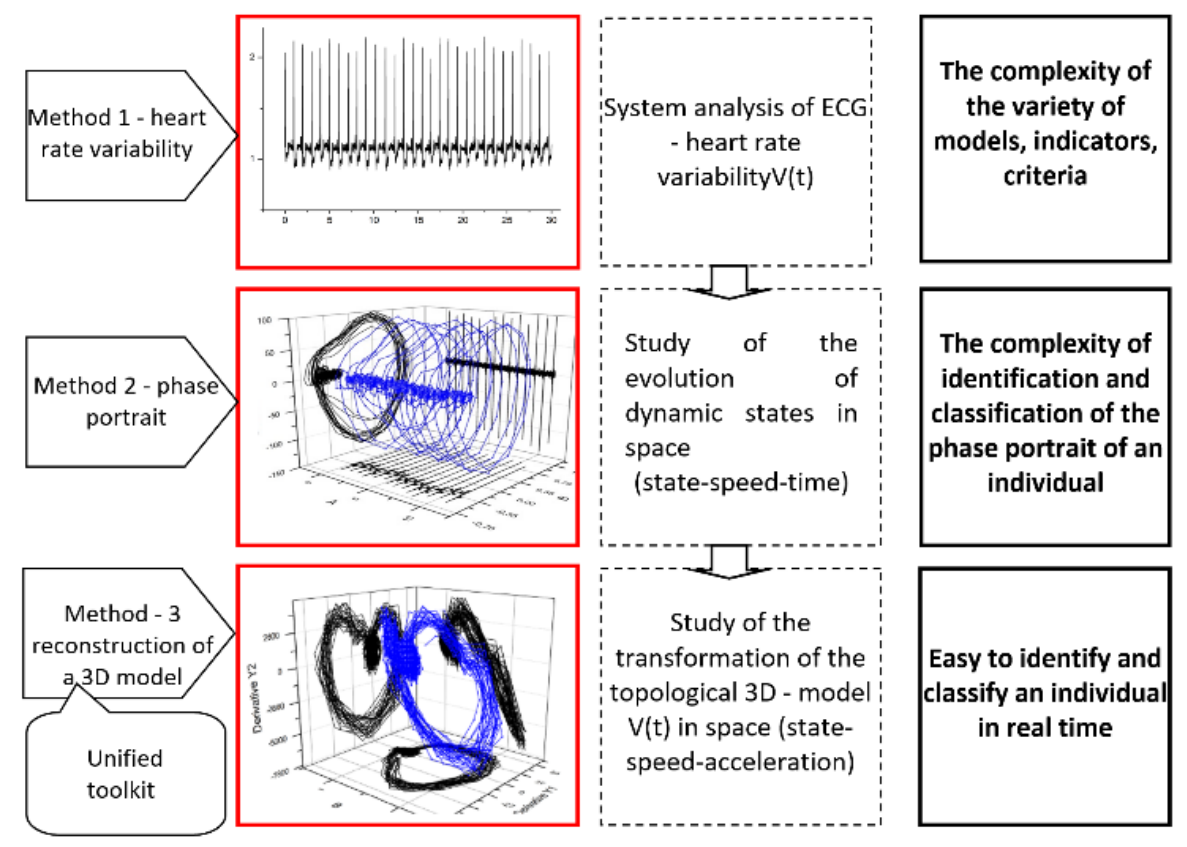

Figure 6. Transformation of the time series $X(t)$ into $2 D$ and $3 D$ functioning model 
The holistic perception of the 3D ECG model and the configurations of its signatures as a set of opposite components gives cognitive information. In particular, the ratio of these components reflects the dynamic, energetic and structural features of functioning. This is because:

(1) morphologically different dynamical systems are functionally subordinated to the same principles of physics (biophysics) and biomimicry;

(2) different dynamical systems operate according to the same thermodynamics laws $[35,36]$;

(3) dynamic processes of different scales s are subordinated the principle of detailed balance.

The character of the restructuring of the signature configuration reflects the change in the structure of the functioning cycle. It allows applying flexible logic of antonyms to:

(1) configuration (form) of signatures (symmetry/asymmetry);

(2) trajectory densities (homogeneous/non-homogeneous) in the signature package;

(3) area covered by the signature (large/small).

Cognitive visualisation gives a holistic perception of dynamic complexity in its versatility and information content. Indicators of dynamic balance and statistical ordering of opposing components provide complementary information. This simplifies the comparison of information flows of different nature and the selection of relevant information sources.

From the analysis of Figure 6 it follows that the causal relationships of the opposite components of the 1st and 2nd orders of signatures can be analyzed from three angles of view in real time. It is the spatio-temporal relationship of dynamic parameters that determines the features of functioning. Consequently, cognitive information is most manifested in the spatio-temporal ordering of the topological 3D model of the time series. Therefore, signatures of the 1 st and 2 nd orders are so informative, changes in the configuration of which can be analyzed by deterministic methods, and the change in their areas can be estimated by statistical methods [32-34, 37, 38].

The geometrisation of functioning (evolution) dynamics of CDS elements allows predicting their viability. Based on the atlas of topological 3D models of functioning and their projections, it is possible to develop an interdisciplinary structural-functional approach to identifying risks, the precursors of which are the transitional functional conditions of objects of animate and inanimate nature. It is based on the developed convergent approach to the viability of CDS elements in extreme conditions.

\section{DYNAMIC MODEL OF RISK MANAGEMENT}

\subsection{Unification of risk assessment}

The main conceptual models for risk management - the COSO model [39] and the FERMA model [40, 41] set certain standards. These models have different representations of the "ideal" risk management process that are difficult to apply to the developing CDS. Simultaneously, the proposed unified toolkit creates the opportunity of a new approach to the developing of biomimetic models of multifractal objects of various natures. Using the relative indicators of order and balance [42] to assess the dynamic and static complexity, it is possible to identify, categorise and evaluate the detected risks.

The dynamic model of risk management simplifies identifying a risk management strategy to keep aggregate potential risk at a low level. Upon such an analysis of the functioning cycles, the CDS elements become more resistant to unforeseen events, especially to various forms of risks.

Figure 7 shows the systemic interconnections of the three levels of risk management strategies. It is clear that ensuring the safety, reliability of the CDS and increasing its stability is impossible without taking into account the cognitive aspects of the interaction between man-machine-environment at all stages of the CDS life cycle.

\subsection{Risk estimation within the frame of the complexity concept}

Unification of risk estimation means can be realised on the analysis of the evolution of the functioning cycle. The unified toolkit allows creating a risk passport for CDS elements. It contains models and signatures for the functioning of relevant information sources that meet the relevant standards' requirements and recommendations. It will make it possible to assess risks using unified indicators of orderliness and balance of the functioning cycle and the nature of its restructuring under test exposure. Determination and maintenance of the required level of risks will allow for regular updating of the risk register, developing the required standards in risk management, and regular monitoring of the CDS operation in non-standard conditions.

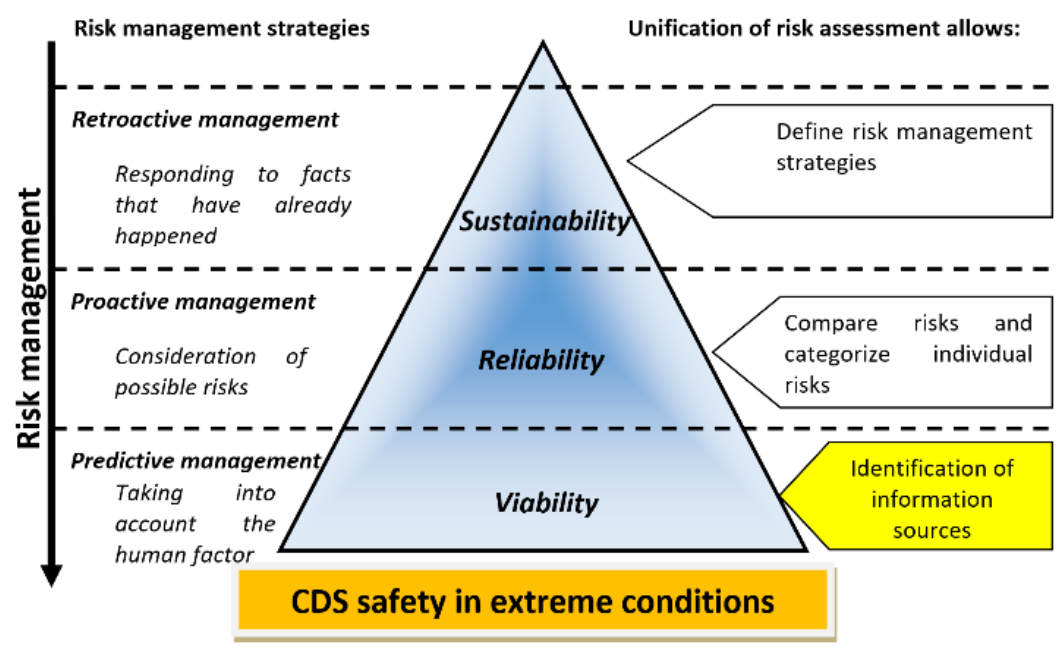

Figure 7. Risk management levels in extreme conditions 


\section{THE APPROACH PROSPECTS AND ITS INNOVATIVE POTENTIAL}

External and internal stressors distort information flows of different nature and create systemic problems. To solve the problems of the viability of CDS elements in extreme conditions, it is necessary to develop interdisciplinary connections during training, as well as to design and operate CDS taking into account the principles of human factors engineering and the corresponding standards. Cognitive visualization of 3D models of fractal signals made it possible to unify the tools for the synthesis and analysis of models of functioning of information sources of different nature. It is based on parametric geometrisation of the spatio-temporal structure of interconnections in the space of dynamic events. The transformation of a fractal signal into a topological 3D model of the functioning and its spatio-temporal signatures makes it possible to assess the complexity in terms of the ordering degree, the energy balance degree using probabilistic and deterministic research methods. The ability to synthesise and analyse CDS models in real-time has high innovative potential.

In particular, the application of the convergent approach and the means for its implementation to information sources of different nature (EMR detector, radiation and acoustic radiation sensors), and to human electrophysiological signals (EEG, EOG, rheogram, etc.) demonstrate advantages. It is established that latent spatio-temporal interconnections determine the viability of the CDS functioning in non-standard conditions. An atlas of these 3D models and their signatures simplifies risk management.

The toolkit and model atlas will also simplify man-machine interactions in the training, design and testing of new CDS. This will increase the safety, reliability and stability of CDS elements functioning under external influences, including the human organism functioning.

\section{CONCLUSIONS}

The digital transformation created systemic latent problems in the security, reliability and resilience of CDS. Their interconnection determines the complexity of solving the viability problem in extreme conditions. Reconstruction of the topological 3D model of the dynamic system functioning according to the measured fractal signal made it possible:

(1) reduce information complexity when modeling CDS functioning;

(2) reveal the latent structure of induced connections determining the individuality of functioning;

(3) evaluate the modelling results visually in real-time that is important for choosing a risk management strategy.

Local distortion of the multifractal signal increases the dynamic and structural complexity. However, the transformation of these distortions into a configuration of ordered opposing components simplifies the cognitive perception of complexity.

It should be noted that the analysis of the topological 3D model of functioning stimulates the active work of both brain hemispheres $[19,38]$ that simplifies cognitive perception and presentation of information flows of different nature. Such visualisation of the CDS elements functioning allows realising the data mining that is not yet available to human and computer in separate. It expands the capabilities of control, machine learning [15, 37], and intelligent support systems [37, 42].

\section{REFERENCES}

[1] Mygal, V., Mygal, G. (2019). Problems of digitized information flow analysis: Cognitive aspects. Information \& Security: An International Journal, 43(2): 134-144. https://doi.org/10.11610/isij.4312

[2] Mygal, G., Mygal, V. (2020). The viability of dynamic systems in difficult conditions: cognitive aspects. In 2020 IEEE 11th International Conference on Dependable Systems, Services and Technologies (DESSERT), pp. 224-229.

https://doi.org/10.1109/DESSERT50317.2020.9125063

[3] Mainzer, K. (1994). Thinking in complexity: The complex dynamics of matter, mind, and mankind. Springer-Verlag Berlin Heidelberg. https://doi.org/10.1007/978-3-662-03014-1

[4] Marquardt, N., Hannig, C., Hannig, S. (2018). The impact of mental readiness on driving performance and traffic safety. International Journal of Safety and Security Engineering, 8(1): 10-19. https://doi.org/10.2495/SAFEV8-N1-10-19

[5] Nicolis, G.; Prigogine, I. (1989). Exploring complexity: An introduction. New York, NY: W.H. Freeman. https://doi.org/10.1063/1.2810725

[6] Beer, S. (1981). Brain of the Firm; Second Edition (much extended), John Wiley, London and New York.

[7] Wiener, N. (1961). Cybernetics: Control and Communication in the Animal and the Machine--2nd. MIT Press.

[8] Prigogine, I. (1993). Chaotic dynamics and transport in fluids and plasmas: Research trends in physics series. New York: American Institute of Physics.

[9] Haken, H. (1996). Principles of brain functioning. A Synergetic Approach to Brain Activity, Behavior and Cognition. Springer-Verlag Berlin Heidelberg. https://doi.org/10.1007/978-3-642-79570-1

[10] Buzan, T., Buzan, B. (2003). Super-Thinking. Popurri Publ.

[11] Russel, J.S., Norvig S. (1995). Artificial Intelligence: A Modern Approach. Prentice-Hall, Inc.

[12] Fedota, J.R., Parasuraman, R. (2010). Neuroergonomics and human error. Theoretical Issues in Ergonomics, 11(5): $402-421$. https://doi.org/10.1080/14639220902853104

[13] Mygal, V.P., Mygal, G.V. (2020). Cognitive and ergonics aspects human interactions with a computer. Radioelectronic and Computer Systems, 1(93): 90-102. https://doi.org/10.32620/reks.2020.1.09

[14] Venda, V.F., Venda, Y.V. (1995). Dynamics in ergonomics, psychology, and decisions: Introduction to ergodynamics. Norwood, NJ: Ablex.

[15] Mygal G., Mygal V. (2018). Interdisciplinary approach to informational teaching environment formation. Odes'kyi Politechnichnyi Universytet Pratsi, 1(54): 9298. https://doi.org/10.15276/opu.1.54.2018.13

[16] Forrester, J.W. (2007). System dynamics-a personal view of the first fifty years. System Dynamics Review, 23(2): 345-358. https://doi.org/10.1002/sdr.382

[17] Schwaninger, M., Rios, J.P. (2008). System dynamics and cybernetics: A synergetic pair. System Dynamics 
Review, 24(2): 145-174. https://doi.org/10.1002/sdr.400

[18] Moore, G.E. (2003). No exponential is forever: But "forever" can be delayed! [semiconductor industry]. In 2003 IEEE International Solid-State Circuits Conference, 2003. Digest of Technical Papers. ISSCC, pp. 20-23. https://doi.org/10.1109/ISSCC.2003.1234194

[19] Mygal, V.P., Mygal, G.V., Balabanova, L.M. (2019). Visualization of signal structure showing element functioning in complex dynamic systems - cognitive aspects. Journal of Nano- and Electronic Physics, 11(2): 02013. https://doi.org/10.21272/jnep.11(2).02013

[20] Мигаль, Г.В., Мигаль, В.П. (2020). Cognitive and ergonomic aspects human interactions with a computer. Radioelectronic and Computer Systems, (1): 90-102. https://doi.org/10.32620/reks.2020.1.09

[21] Evans, D.J., Searles D.J., Mittag, E. (2001). Fluctuation theorem for hamiltonian systems: Le Chatelier's principle. Physical Review E, 63(5): 051105. https://doi.org/10.1103/PhysRevE.63.051105

[22] French, S. (2014). The Structure of the World: Metaphysics and Representation. Oxford University Press.

[23] Mygal, V.P., But, A.V., Mygal, G.V., Klimenko, I.A. (2016). An interdisciplinary approach to study individuality in biological and physical systems functioning. Scientific Reports, 6(1): 1-7. https://doi:10.1038/srep29512

[24] Passino, K.M. (2005). Biomimicry for Optimization, Control, and Automation. Springer-Verlag London. https://doi.org/10.1007/b138169

[25] Bak, P. Tang, C., Wiesenfeld, K. (1987). Self-organized criticality: An explanation of $1 / \mathrm{f}$ noise. Physical Review Letters, 59(4): 381-384. https://doi.org/10.1103/PhysRevLett.59.381

[26] Stöltzner, M. (2003). The principle of least action as the logical empiricist's Shibboleth. Studies in History and Philosophy of Science Part B: Studies in History and Philosophy of Modern Physics, 34(2): 285-318. https://doi.org/10.1016/S1355-2198(03)00002-9

[27] Katzav, J. (2004). Dispositions and the principle of least action. Analysis, 64(3): 206-214. https://doi.org/10.1093/analys/64.3.206

[28] Terekhovich, V. (2018). Metaphysics of the principle of least action. Studies in History and Philosophy of Science Part B: Studies in History and Philosophy of Modern Physics, 62: 189-201. https://doi.org/10.1016/j.shpsb.2017.09.004

[29] Georgiev, G.Y. (2012). Quantitative measure, mechanism and attractor for self-organization in networked complex systems. self-organizing systems. 6th IFIP TC 6 International Workshop, Delft, The Netherlands, pp. 90-95. https://doi.org/10.1007/978-3642-28583-7 9

[30] Edwards, A.W.F. (2007). Maximisation principles in evolutionary biology. Philosophy of Biology, 335-347. https://doi.org/10.1016/B978-044451543-8/50017-4

[31] Mygal, V.P., But, A.V., Phomin, A.S., Klimenko, I.A. (2015). Geometrization of the dynamic structure of the transient photoresponse from zinc chalcogenides. Semiconductors, $\quad 49(5)$ : 634-637. https://doi.org/10.1134/S1063782615050152

[32] Mygal, V.P., Klymenko, I.A., Mygal, G.V. (2017). Individuality of photoresponse dynamics of semiconductor sensors. Functional Materials, 24(2): 212-

218. https://doi.org/10.15407/fm24.02.212

[33] Mygal, V.P., Klymenko, I.A., Mygal, G.V. (2018). Influence of radiation heat transfer dynamics on crystal growth. Functional Materials, 25(3): 574-580. https://doi.org/10.15407/fm25.03.574

[34] PhysioNet. The research Resource for Complex Physiologic Signals. http://physionet.org, accessed on 6 December 2020.

[35] Maugin, G.A. (1988). Continuum mechanics of electromagnetic solids. North Holland. https://doi.org/10.1115/1.3176205

[36] Yariv, A., Yen, P. (2002). Optical Waves in Crystals: Propagation and Control of Laser Radiation. A WileyInterscience Publication. John Wiley and Sons. NewYork.

[37] Mygal, V., Mygal, G., Chukhray, A., Havrylenko, O. (2020). Application of space-time patterns in tutoring. In ICTERI., pp. 430-437.

[38] Mygal, V.P., Mygal, G.V. (2020). Convergent approach to identification of transient states of a dynamic system. Nano-Electron. $\quad$ Phys., $12(6)$ : 06018. https://doi.org/10.21272/jnep.12(6).06018

[39] Steinberg, R.M., Martens, F.J., Everson, M.E.A., Nottingham L.E. (2004). Enterprise risk management integrated framework. Executive Summary. The Committee of Sponsoring Organizations of the Treadway Commission, COSO. Altamonte Springs, Florida, USA.

[40] A Risk Management Standard (2002). The Association of Insurance and Risk Managers (AIRMIC). The National Forum for Risk Management ALARM, the Institute of Risk Management (IRM), Brussels, Belgium.

[41] ISO 31000. (2018). Risk management - Guidelines. https://www.iso.org/standard/65694.html.

[42] Mygal, V.P., Mygal, G.V., Illiashenko, O. (2021). Intelligent decision support - cognitive aspects. Digital Transformation, Cyber Security and Resilience of Modern Societies, 84: 395.

\section{NOMENCLATURE}

$\begin{array}{ll}\text { Abbreviations } & \\ \text { 3D model } & \text { three-dimensional model } \\ \text { EEG } & \text { electroencephalogram } \\ \text { ECG } & \text { electrocardiogram } \\ \text { EOG } & \text { electrooculogram } \\ \text { CDS } & \text { complex dynamic systems } \\ \text { RAM } & \text { random access memory } \\ \text { EMR } & \text { electromagnetic radiation }\end{array}$

\section{Symbols}

D

$\mathrm{H}_{\text {Sh }}$

$\mathrm{H}_{\mathrm{K}}$

$\mathrm{H}_{\mathrm{R}}$

$\mathrm{K}$

L

$\mathrm{T}$

$\mathrm{V}(\mathrm{t})$

$X(t)$

$\mathrm{dX}(\mathrm{t}) / \mathrm{dt}$

$\mathrm{d}^{2} \mathrm{X} / \mathrm{dt}^{2}$ fractal dimension

Shannon's entropy

Kolmogorov's entropy

entropy Rainier

integer

length

time

electrophysiological signal

state-coordinate

speed-slope

acceleration-curvature 